\title{
A numerical study on the estimation of the stable size distribution for a cell population balance model
}

\section{Luis M. Abiab ${ }^{b}$ Óscar Angulo ${ }^{a *}$, Juan Carlos López-Marcos ${ }^{b}$ and Miguel Ángel López-Marcos ${ }^{b}$}

The presence of a steady-state distribution is an important issue in the modelization of cell populations. In this paper, we analyse, from a numerical point of view, the approach to the stable size distribution for a size-structured balance model with an asymmetric division rate. To this end, we introduce a second order numerical method based on the integration along the characteristic curves over the natural grid. We validate the interest of the scheme by means of a detailed analysis of convergence. Copyright (C) 0000 John Wiley \& Sons, Ltd.

Keywords: cell population balance models; asymmetric division; numerical methods; natural grid; convergence analysis; stable size distribution

\section{Introduction}

We consider the numerical integration of a model which is based on the one proposed by Ramkrishna [19]. It describes the evolution of a size-structured cell population and takes the following form

$$
\begin{aligned}
u_{t}(x, t)+(g(x) u(x, t))_{x} & =-\mu(x) u(x, t)-b(x) u(x, t)+2 \int_{x}^{1} b(s) P(x, s) u(s, t) d s, \quad x_{\min }<x<1, t>0 \\
u\left(x_{\min }, t\right) & =0, \quad t>0 \\
u(x, 0) & =\varphi(x), \quad x_{\min } \leq x \leq 1 .
\end{aligned}
$$

The independent variables $x$ and $t$ represent size and time respectively, $x_{\min }$ stands for a nonnegative minimum cell-size and we consider a maximal cell-size, normalized to 1 . The dependent variable $u(x, t)$ is the size-specific density of cells with size $x$ at time $t$. The size of any cell varies according to the following ordinary differential equation

$$
\frac{d x}{d t}=g(x)
$$

a Departamento de Matemática Aplicada e IMUVA. ETSIT. Universidad de Valladolid. 47011 Valladolid. SPAIN.

b Departamento de Matemática Aplicada e IMUVA. Facultad de Ciencias. Universidad de Valladolid. 47011 Valladolid. SPAIN.

* Correspondence to: Óscar Angulo, Departamento de Matemática Aplicada, ETSIT, Pso. Belén 5, 47011 Valladolid. SPAIN. E-mail: oscar@mat.uva.es 
where $g(x)$ is the growth rate with $g>0$ on $\left[x_{\min }, 1\right)$. The nonnegative functions $\mu$ and $b$ represent the mortality and division rates respectively. These are usually called the vital functions and define the life history of cells. Finally, the distribution of sizes between the two daughter cells at the moment of cell division (unequal division) is defined in terms of a conditional density $P(x, y)$, called the partitioning function, which gives the distribution of the size of a daughter-cell $x$, when the size of the mother is equal to $y$. Thus, $\int_{x_{1}}^{x_{2}} P(x, y) d x$ means the probability for a daughter cell to have size $x$ in the interval $\left(x_{1}, x_{2}\right)$ knowing that the mother had size $y$. Such distribution should verify the following properties:

$$
\int_{x_{\min }}^{1} P(x, y) d x=1, \quad P(x, y)=P(y-x, y), \quad P(x, y)=0, x \geq y .
$$

As a particular (and extreme) case, mother cells could always divide into two identical daugther cells (equal division). In that case, the partitioning function reduces to the Dirac delta function $P(x, y)=\delta(x-y / 2)$, and it turns into the model proposed by Diekmann et al. [8]. In these models, the environment is assumed to have unlimited space and resources.

A remarkable feature is the existence of a maximum individual size. This is a biological truth: cells must divide or die before reaching this value. Therefore, $\Pi(1)=0$ where

$$
\Pi(x)=\exp \left(-\int_{x_{\min }}^{x} \frac{\mu(s)+b(s)}{g(s)} d s\right), \quad x_{\min } \leq x \leq 1
$$

which represents the probability that an individual of size $x_{\min }$ reaches size $x$. If the division and mortality rates are bounded functions, cells will not disappear at the maximum size and the previous condition cannot be satisfied unless they do not reach such a value. For this purpose, we consider a growth function that verifies $\lim _{x \rightarrow 1^{-}} \int_{x_{\min }}^{x} \frac{d s}{g(s)}=+\infty$. Note that this hypothesis implies $g(1)=0$ if $g$ is a continuous function defined in $\left[x_{\min }, 1\right]$. Henceforth, in the present model, we assume that cell-size is strictly increasing during lifetime of cells and always less than one. Moreover, if we assume that initially there are no cells of maximum size, the solution to the problem satisfies $u(1, t)=0, t>0[7]$.

Cell population balance models were introduced in the early 1960s within the framework of particle dynamics in chemical and cellular contexts $[21,5,11]$. Despite this early development, nowadays it is an area of increasing applications and it is used to describe quite different issues (see [20] and references therein). In recent years, they have evolved towards more complicated models: several structuring variables, various populations (describing, for example, proliferating and quiescent cells or the different stages in a cell-cycle), nonlinear problems (with the consumption of a limited extracellular medium), inverse problems to compute the vital functions $[6,9,10,22]$. In our setting, we consider a cell population balance model structured by the cell-size in which the reproduction is carried out by fission into two daughter cells with different sizes. Cell-size is an attractive variable as a result of the relative ease and precision with which it can be measured because the instrumentation to obtain it has improved considerably. The model that arises because of this simplification is still useful in order to analyse and understand cell population dynamics.

From a theoretical point of view, a general survey of the main mathematical problems solved and the principal techniques employed in this context is given in $[17,16,4,18]$. Properties such as existence, uniqueness, etc., could be studied without an explicit expression for the solution. However, the knowledge of their qualitative or quantitative behaviour in a more tangible way sometimes becomes necessary. Therefore, numerical methods provide a valuable tool to obtain such information.

One of the most important issues in the formulation of cell population balance models from both a qualitative and quantitative point of view is to establish the existence of a size pyramid. The main purpose in the study of system (1.1)-(1.3) has been to set the experimental evidence of a stable size distribution. A size distribution is represented as

$$
\frac{u(x, t)}{\int_{x_{\min }}^{1} u(x, t) d x}
$$

A stable steady distribution is achieved if there exists a function $\pi(x)$ such that the size distribution tends to $\pi(x)$, as time $t$ tends to $+\infty$. The existence of $\pi$ implies a solution of (1.1)-(1.3) of the type $C e^{\sigma t} \pi(x)$ where $\sigma$ is an asymptotic value of the relative growth rate of the population, when it exits it is unique and known as the Malthusian parameter of the problem, and only $C$ depends on the initial condition. An interesting property of the stable size distribution is that whatever the initial distribution 
may be, it is shaped asymptotically as the function $u^{*}$, this property is called asynchronicity. Even if the initial distribution has a very narrow support, it will tend to occupy asymptotically the whole support of $\pi$, therefore we can generate a large population (a clone) from a single cell. From a theoretical point of view, several authors have proved the existence of stable size distribution with different hypotheses $[8,13]$.

From a numerical point of view, in the last twenty years, several studies have addressed their numerical solution with different techniques: analytical solutions based on a successive generations approach, classical finite difference schemes, finite element or spectral methods or the use of the integration along the characteristics (see [2] and references therein). However, the analysis of most of these numerical proposals is not finished yet and its convergence to the theoretical solution has been ensured only in a few of them [3]. In the present paper, we present a second-order characteristics method, based on the numerical scheme developed and analysed in [3] for the symmetric division case. Second-order methods maintain a good compromise between the required smoothness of the vital functions based on realistic biological data and the efficiency of the numerical schemes. We employ a numerical approximation to an invariant grid on the state variable and the discretization of the integral representation of the solution to the problem along the characteristic curves. We also prove the optimal rate of convergence under appropriate regularity assumptions.

The paper is organized as follows. In Section 2, we introduce the proposed numerical method. Section 3 is devoted to a representative numerical simulation which shows how we use the new method to approximate the stable size distribution of the model. Finally, we strengthen the rationale for the scheme with an appendix where we analyse the convergence of the numerical solution.

\section{Numerical Method}

We rewrite (1.1) as

$$
u_{t}(x, t)+g(x) u_{x}(x, t)=-\mu^{*}(x) u(x, t)+2 \int_{x}^{1} b(s) P(x, s) u(s, t) d s, \quad x_{\min }<x<1, t>0
$$

where we have defined $\mu^{*}(x)=g^{\prime}(x)+\mu(x)+b(x)$. We denote by $x\left(t ; t_{*}, x_{*}\right)$ the characteristic curve of the equation (2.1) (and (1.1)) that takes the value $x_{*}$ at the time instant $t_{*}$, and define $w\left(t ; t_{*}, x_{*}\right)=u\left(x\left(t ; t_{*}, x_{*}\right), t\right), t \geq t_{*}$. Thus,

$$
\left\{\begin{array}{l}
\frac{d}{d t} x\left(t ; t_{*}, x_{*}\right)=g\left(x\left(t ; t_{*}, x_{*}\right)\right), \quad t>t_{*} \\
x\left(t_{*} ; t_{*}, x_{*}\right)=x_{*}
\end{array}\right.
$$

and

$$
\left\{\begin{array}{l}
\frac{d}{d t} w\left(t ; t_{*}, x_{*}\right)=-\mu^{*}\left(x\left(t ; t_{*}, x_{*}\right)\right) w\left(t ; t_{*}, x_{*}\right)+2 \int_{x\left(t ; t_{*}, x_{*}\right)}^{1} b(s) P\left(x\left(t ; t_{*}, x_{*}\right), s\right) u(s, t) d s, \quad t>t_{*}, \\
w\left(t_{*} ; t_{*}, x_{*}\right)=u\left(x_{*} \cdot t_{*}\right)
\end{array}\right.
$$

We will obtain a numerical approximation to the solution $u$ of (2.1) and (1.2)-(1.3) on a fixed time interval [0,T]. The numerical method comprises two basic steps. The first one is to build a grid $\left\{\left(x_{j}, t^{n}\right): 0 \leq j \leq J+1,0 \leq n \leq N\right\}$, on $\left[x_{\min }, 1\right] \times[0, T]$, with $x_{\min }=x_{0}<x_{1}<\cdots<x_{\jmath}<x_{\lrcorner+1}=1$, such that points $\left(x_{j}, t^{n}\right)$ and $\left(x_{j+1}, t^{n+1}\right), 0 \leq j \leq J+1,0 \leq n \leq N$, belong to the same characteristic curve. This time-invariant grid is usually known as the natural grid and was first introduced in [15]. Its invariance allows us to study the long-term behaviour of the model which has proven to be one of the easiest ways to discretise the population states (cell-sizes). With this aim, we solve numerically (2.2) with an integrator for ordinary differential equations. The second step is to compute an approximation to $u\left(x_{j+1}, t^{n+1}\right)$ starting from a numerical approach to $u\left(x_{j}, t^{n}\right)$. To this end, we discretize the solution to (2.3), which is given by

$$
\begin{aligned}
w\left(t ; t_{*}, x_{*}\right)= & u\left(x_{*}, t_{*}\right) \exp \left\{-\int_{t_{*}}^{t} \mu^{*}\left(x\left(\tau ; t_{*}, x_{*}\right)\right) d \tau\right\} \\
& \left.+2 \int_{t_{*}}^{t} \exp \left\{-\int_{\tau}^{t} \mu^{*}\left(x\left(s ; t_{*}, x_{*}\right)\right) d s\right\}\left(\int_{x\left(\tau ; t_{*}, x_{*}\right)}^{1} b(\sigma) P\left(x ; t_{*}, x_{*}\right), \sigma\right) u(\sigma, \tau) d \sigma\right) d \tau, \quad t \geq t_{*} .
\end{aligned}
$$


This approximation technique has been applied to different models in [2] and [3]. On the one hand, a useful first-order scheme was proposed to obtain the solution to a nonlinear generalization of (1.1)-(1.3) when the vital functions involved in the problem depend on an abiotic environment that changes with time [2]. It is known that low-order of convergence would produce a lack of efficiency which could be reduced with higher order methods. However, the smoothness of the solution to (1.1)-(1.3) is not as high as these last schemes demand. Thus, second-order methods present a good balance: they enhance the efficiency even with a lack of regular data. On the other hand, a novel second-order method was introduced for the equal fission model in [3]. In this work, we present an adaptation of this method to the more general asymmetric division case.

\subsection{The grid points}

Let $N$ be a positive integer. We define $k=\frac{T}{N}$ and introduce the discrete time levels $t^{n}=n k, 0 \leq n \leq N$. As we have mentioned, we want to introduce a state variable grid

$$
x_{\min }=x_{0}<x_{1}<\cdots<x_{\lrcorner}<x_{\lrcorner+1}=1,
$$

such that points $\left(x_{j}, t^{n}\right)$ and $\left(x_{j+1}, t^{n+1}\right), 0 \leq j \leq J+1,0 \leq n \leq N$, belong to the same characteristic curve. This grid is nonuniform and invariant with time because the growth rate function is, explicitly, independent of the time variable. We want to stress that $J$ is an a posteriori chosen integer and represents the last grid point computed. In general, we are unable to solve (2.2) analytically, hence we integrate it numerically. We consider the grid points defined by the equations

$$
x_{0}=x_{\min } ; \quad x_{j+1}=x_{j}+\frac{k}{2}\left(g\left(x_{j}\right)+g\left(x_{j}+k g\left(x_{j}\right)\right)\right), \quad 0 \leq j \leq J-1 ; \quad x_{\jmath+1}=1 ;
$$

where we employ the modified Euler method, a second order Runge-Kutta method. In [1], it is stated that we need the continuity and positivity of function $g$ on $[0,1)$ to satisfy $(2.5)$ and ensure the existence of $x_{\jmath}$, such that $K_{0} k<1-x_{\lrcorner} \leq K_{1} k$, with $K_{0}$ and $K_{1}$ suitable constants. It also establishes estimates for the local error when $g$ is smooth enough. Note that actually the points $\left(x_{j}, t_{n}\right)$ and $\left(x_{j+1}, t_{n+1}\right), 0 \leq j \leq J-1,0 \leq n \leq N-1$, belong to the same numerical characteristic curve.

\subsection{Numerical integration along the characteristics}

We refer to the grid point $x_{j}$ by a subscript $j$ and to the time level $t^{n}$ by a superscript $n$. Let $U_{j}^{n}$ be a numerical approximation to $u_{j}^{n}=u\left(x_{j}, t^{n}\right), 0 \leq j \leq J-1,0 \leq n \leq N-1$. The next stage is to propose a one-step method in order to obtain an approximation $U_{j+1}^{n+1}$ to $u_{j+1}^{n+1}$. To this end, we use, with step size $k$, the following second-order discretization of (2.4). For $0 \leq n \leq N-1$,

$$
U_{j+1}^{n+1}=\exp \left\{-\frac{k}{2}\left(\mu^{*}\left(x_{j}\right)+\mu^{*}\left(x_{j+1}\right)\right)\right\}\left(U_{j}^{n}+k \mathcal{Q}_{k}^{j}\left(\mathbf{b} \cdot \mathbf{P}^{j} \cdot \mathbf{U}^{n}\right)\right)+k \mathcal{Q}_{k}^{j+1}\left(\mathbf{b} \cdot \mathbf{P}^{j+1} \cdot \mathbf{U}^{n+1}\right), \quad 0 \leq j \leq J-1 .
$$

In this formula, $\mathcal{Q}_{k}^{\prime}(\mathbf{V})$ represents a quadrature rule to approximate the integral over the interval $\left[x_{l}, 1\right], 0 \leq I \leq J$, of the function with grid values $\mathbf{V}=\left[V_{0}, \ldots, V_{J+1}\right]$. In this case $\mathbf{b}, \mathbf{P}^{\prime}$ and $\mathbf{U}^{m}$, represent the vectors with components $\left[b\left(x_{0}\right), \ldots, b\left(x_{J+1}\right)\right]$, $\left[P\left(x_{l}, x_{0}\right), \ldots, P\left(x_{l}, x_{J+1}\right)\right]$ and $\left[U_{0}^{m}, \ldots, U_{J+1}^{m}\right]$, respectively, and products $\mathbf{b} \cdot \mathbf{P}^{\prime} \cdot \mathbf{U}^{m}, 0 \leq I \leq J, 0 \leq m \leq N$, must be interpreted component-wise. The approximating values at the minimum and maximum sizes are

$$
U_{0}^{n+1}=U_{J+1}^{n+1}=0
$$

With regard to the nonlocal terms, we consider the composite trapezoidal quadrature rule on the grid inside the interval $\left[x_{l}, 1\right]$

$$
\mathcal{Q}_{k}^{\prime}(\mathbf{V})=\sum_{j=l}^{J} \frac{x_{j+1}-x_{j}}{2}\left(V_{j}+V_{j+1}\right), \quad I=0, \ldots, J
$$

The numerical procedure seems to be implicit. However, if we compute the approximations at the new time level $t^{n+1}$ downwards (that is, first $U_{J+1}^{n+1}$ using (2.8), then $U_{j+1}^{n+1}$ from $J-1$ to 0 using (2.7), and finally $U_{0}^{n+1}$ using (2.8) again), it results in an explicit procedure. 
Assuming suitable regularity conditions in the vital functions and the solution to problem (1.1)-(1.3), we can prove the second order convergence of the numerical approximation to the exact solution $u$. We want to emphasise that the number of nodes in the natural grid is not determined with respect to $N$ (and therefore, with respect to $k$ ), but even so we obtain the convergence of the quadrature rule under this premise. In [1], conversely, a subgrid of the natural grid is introduced to overcome this difficulty. In Appendix A, we provide the demonstration based on the consistency property of the method. This result has been validated by means of an extensive numerical simulation carried out with different text problems, final-times $T$ and step-sizes $k$, not included in this paper.

\section{Numerical study on the Stable Size distribution}

Taking into account the interest of the discretization method in the numerical approximation of problem (1.1)-(1.3), we use it for the analysis of the associated stable size distribution. The model has a stable size distribution if there exists a function $\pi(x)$ and a value $\sigma$ such that

$$
\sigma \pi(x)=-(g(x) \pi(x))^{\prime}-(\mu(x)+b(x)) \pi(x)+2 \int_{x}^{1} b(s) P(x, s) \pi(s) d s, \quad \pi\left(x_{\min }\right)=0 .
$$

And, if we denote

$$
P(t)=\int_{x_{\min }}^{1} u(x, t) d x
$$

the function $u(x, t) / P(t)$ tends to $\pi(x)$, as time $t$ tends to $+\infty$. The existence of a distribution $\pi(x)$ implies the existence of a solution of (1.1)-(1.3) of the type $e^{\sigma t} \pi(x)$. The number $\sigma$ is unique and known as the malthusian parameter of system (1.1)(1.3). A remarkable feature is the asynchonicity [4], i.e., it is possible to start from a single cell and generate a large population. The existence of a stable-size distribution for our problem could be studied in two different ways following the techniques employed in $[13,12,14]$. Firstly, by means of a change of variable that transforms the problem into an age-structured one. Secondly, with the use of specific division rate functions as, for example, (3.10).

The following simulation shows the appearance of the asynchronous exponential growth with one of the experiments introduced in [2]. We introduce a minimum size $a$ at which a cell divides, $x_{\min } \leq a<1$, to incorporate a more realistic behaviour. So, we assume that the division rate $b$ vanishes at the interval $\left[x_{\min }, a\right]$. We consider $x_{\min }=0, a=\frac{1}{4}, \mu(x)=0, g(x)=0.1(1-x)$. We use the size-specific division rate function

$$
b(x)=\left\{\begin{array}{l}
0, \text { if } \quad x \in\left[0, \frac{1}{4}\right], \\
g(x) \frac{\phi_{b}(x)}{1-\int_{1 / 4}^{x} \phi_{b}(s) d s}, \text { if } \quad x \in\left[\frac{1}{4}, 1\right],
\end{array}\right.
$$

where we have considered that each cell has a stochastically predetermined size at which fission has to occur, which is given by a probability density $\phi_{b}[17]$. In this case

$$
\phi_{b}(x)=\lambda\left\{\begin{array}{l}
\left(x-\frac{1}{4}\right)^{3}, \text { if } \quad x \in\left[\frac{1}{4}, \frac{5}{8}\right] \\
\frac{459}{4096}-\frac{9}{4}\left(x-\frac{13}{16}\right)^{2}+16\left(x-\frac{13}{16}\right)^{4}, \text { if } x \in\left[\frac{5}{8}, 1\right]
\end{array}\right.
$$

and $\lambda=\frac{81920}{3159}$. We take the same partitioning function as in [2],

$$
P(x, y)=\left\{\begin{array}{l}
\frac{1}{\beta(40,40)} \frac{1}{y}\left(\frac{x}{y}\right)^{39}\left(1-\frac{x}{y}\right)^{39}, \text { if } \quad x<y \\
0, \text { if } \quad x \geq y
\end{array}\right.
$$

where $\beta(x, y)$ is the classical Euler beta function. Finally, the initial condition will be chosen to avoid discontinuities caused by an incompatibility with the boundary one. 
Hence, we compute an approximation to the stable size distribution by using the numerical solution obtained with the numerical method. We describe the evolution of the size-distribution with a numerical counterpart of (1.4), that is

$$
\frac{U_{j}^{n}}{\mathcal{Q}_{k}^{0}\left(\mathbf{U}^{n}\right)}, \quad 0 \leq j \leq J+1
$$

that will converge to $U_{j}^{*}, 0 \leq j \leq J+1$, an approximation of the stable size distribution if it exists. We observe that a good approximation to the stable size distribution is reached with moderate values of $T$ (we observe that $T=100$ is enough). Once we reach such values, assuming $U_{j}^{n} \approx C e^{\sigma t^{n}} U_{j}^{*}$, we estimate $\sigma$ by means of the comparison of consecutive time steps (using the total populations, for instance) and $C$.

First, we show how the problem reaches the stable size distribution. We have made an extensive numerical experimentation with different initial conditions with the same conclusion but we show the results we obtain with a compatible initial condition with some oscillations which could have introduced a different behaviour,

$$
\varphi(x)=\left\{\begin{array}{l}
0, \text { if } \quad x \in\left[0, \frac{1}{8}\right] \\
\varphi_{1}\left(x-\frac{1}{8}\right)^{3}(1-x)(\sin (m \pi x+\pi)+1), \text { if } \quad x \in\left[\frac{1}{8}, 1\right]
\end{array}\right.
$$

( $m$ is an integer, the higher the larger number of oscillations, and coefficient $\varphi_{1}$ is chosen in order to assure that the maximum value of $\varphi(x)$ is 1 ). We have employed different values of $m$ but, in Figure 1 , we present the simulation with step-size $k=0.01$ and $m=20$. In the left-hand picture, we show the evolution of the size distribution and, in the right-hand picture, the arising approximation to the stable size distribution. The estimated values of the parameters are $\sigma=6.151916 \mathrm{e}-2$ and $C=2.205377$.
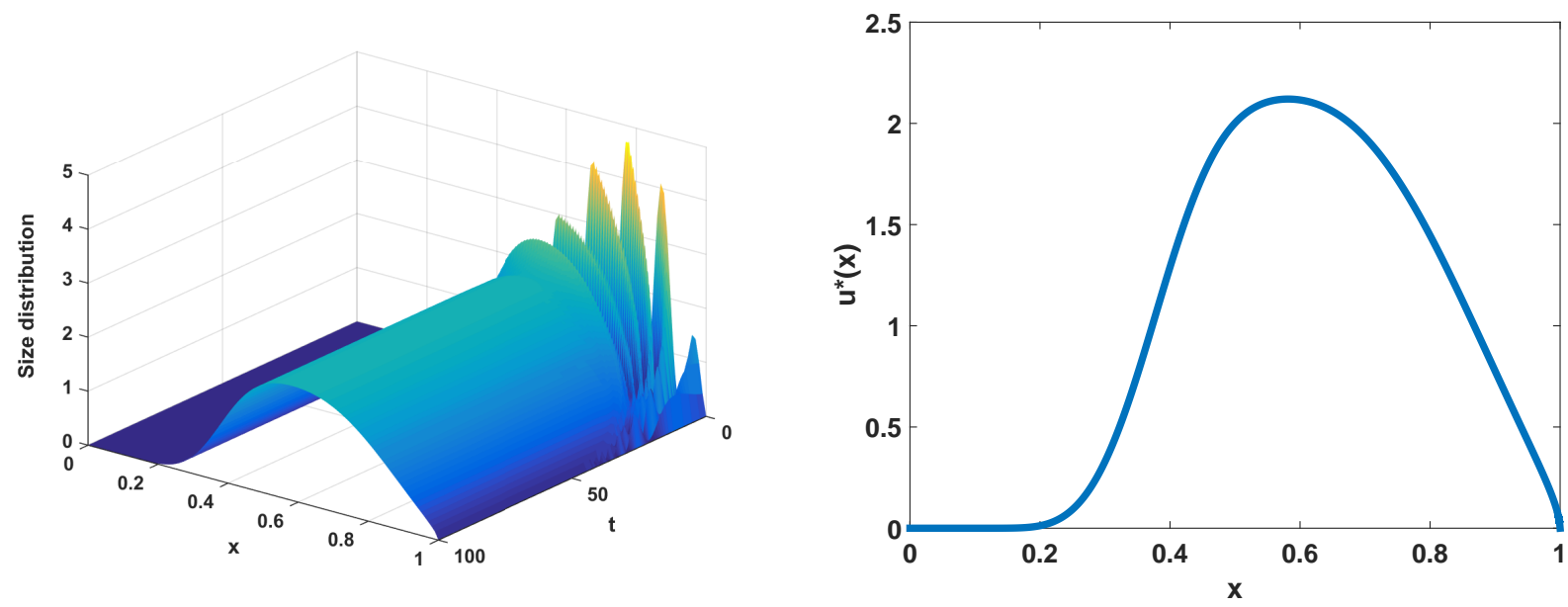

Figure 1. Left-hand picture: Evolution of the size distribution. Right-hand picture: Numerical stable size distribution $u^{*}$ with division rate $b$ given in (3.10)

In order to analyse the behaviour of the solution depending on the initial distribution, we have checked with various initial conditions, however here we present only the results obtained with

$$
\varphi_{\alpha}(x)=\left\{\begin{array}{l}
0, \text { if } \quad x \in\left[0, \frac{1}{8}\right], \\
\alpha \varphi_{1}\left(x-\frac{1}{8}\right)^{3}(1-x), \text { if } \quad x \in\left[\frac{1}{8}, 1\right],
\end{array}\right.
$$

( $\alpha \in \mathbb{R}$, coefficient $\varphi_{1}$ is chosen in order to assure that the maximum value of $\varphi(x)$ is $\alpha$ ). Of course, in all cases, we obtain the same numerical stable size distribution as presented in Figure 1 (right-hand picture). Table 1 shows the computed values of $C$ and $\sigma$ for different values of $\alpha$. We clearly observe the independence of the initial data of the Malthusian parameter, while the constant $C$ shows its dependence on it: $C$ is multiplied by the same factor as the initial data.

Now, we are interested to know the influence on the stable size distribution due to changes in data functions. As we pointed out previously, the existence of a unique stable size distribution has been established theoretically. Thus any change to the 


\begin{tabular}{c||c|c|}
$\alpha$ & $C$ & $\sigma$ \\
\hline \hline 1 & $4.408119 \mathrm{e}-1$ & $6.151916 \mathrm{e}-2$ \\
2 & $8.816239 \mathrm{e}-1$ & $6.151916 \mathrm{e}-2$ \\
4 & $1.763248 \mathrm{e} 0$ & $6.151916 \mathrm{e}-2$ \\
8 & $3.526495 \mathrm{e} 0$ & $6.151916 \mathrm{e}-2$ \\
\hline
\end{tabular}

Table 1. Computed values $C$ and $\sigma$ for different initial conditions $\varphi_{\alpha} . T=100$.

data functions will modify it and, likewise, the Malthusian parameter and the constant $C$. With this purpose, we use again the following data input: $x_{\min }=0, a=\frac{1}{4}, \mu(x)=0, g(x)=0.1(1-x), P(x, y)$ and the initial data as given in (3.11) and (3.12), $\alpha=1$, respectively, but now the size-specific division rate is modified as follows,

$$
b_{\gamma}(x)=b_{1}^{\gamma}\left(x-\frac{1}{4}\right)^{3}(1-x)^{\gamma}, \quad \frac{1}{4} \leq x \leq 1, \quad \gamma \geq 0
$$

(coefficient $b_{1}^{\gamma}$ is chosen in order to assure that the maximum value of $b_{\gamma}(x)$ is 1 ). As parameter $\gamma$ grows, the maximum value in the division rate is reached at smaller sizes and, then, we expect that the density of larger cells decreases.

The change in the division rate, which we have plotted on the right-hand picture of Figure 2 for different values of $\gamma$, implies significant differences in the arising steady state (left-hand picture of Figure 2). We observe how the increase of parameter $\gamma$ affects its shape. This influence appears at the locus and value of the maximum of the stable size distribution and, also, at significant densities in the size variable. When the parameter $\gamma$ induces an earlier division, the maximum of the stable size distribution increases and it is obtained at a smaller size (it seems to be at the half value of the corresponding maximum locus in the division rate). Consequently, the domain given by significant densities decreases.
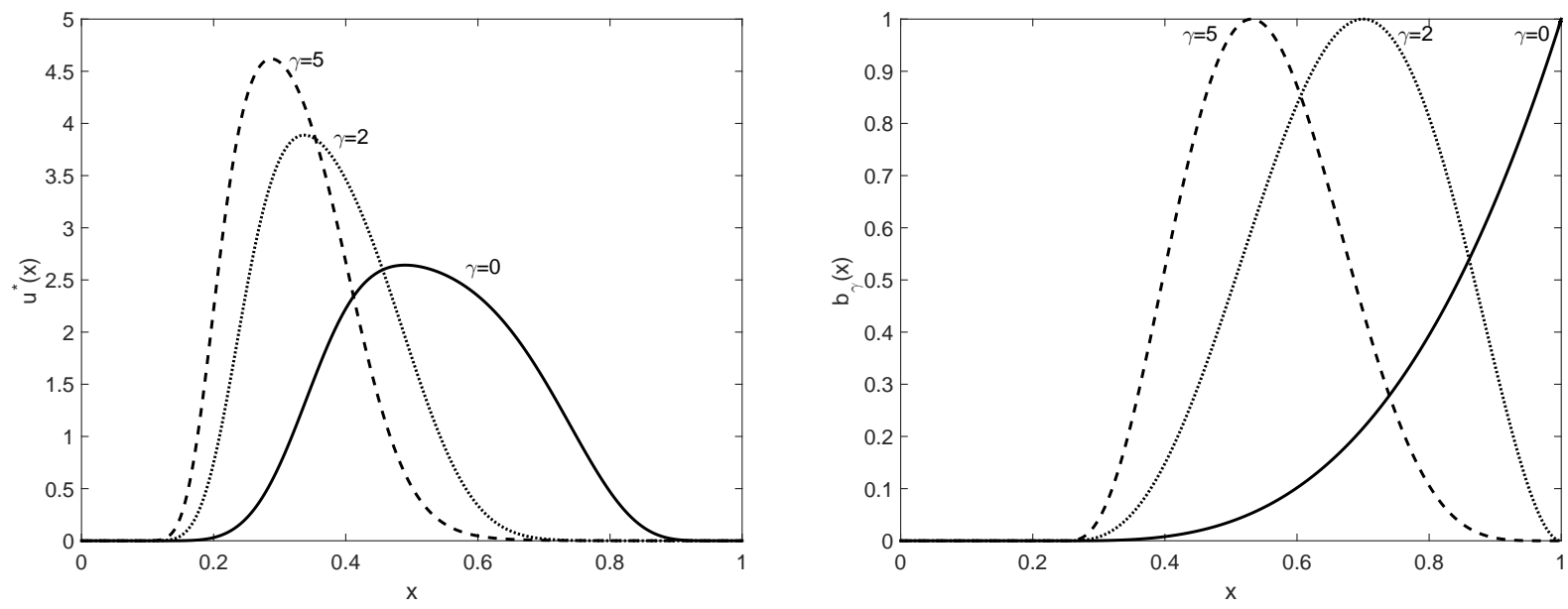

Figure 2. Stable size distribution (left-hand picture) for different division rates $b_{\gamma}$ (right-hand picture).

Finally, in Table 2, we display the computed values of $\sigma$ and $C$ for different values of $\gamma$. We observe, as expected, different values of $\sigma$ for different values of $\gamma$.

\section{Conclusions}

This work focuses on a population balance model applied to cell dynamics. Nowadays, this is an area in which an increasing number of applications are widely used. We consider a cell-size structured population model that describes the dynamics of a cell population when the reproduction process is achieved by division in two unequal parts. We provide a numerical approximation to the analytical solution of the problem because it is difficult to obtain in a general situation. There is a lack of numerical methods 


\begin{tabular}{c||c|c|}
$\gamma$ & $C$ & $\sigma$ \\
\hline \hline 0 & $5.252692 \mathrm{~d}-1$ & $8.823196 \mathrm{~d}-2$ \\
1 & $6.285650 \mathrm{~d}-1$ & $1.408027 \mathrm{~d}-1$ \\
2 & $6.082363 \mathrm{~d}-1$ & $1.680984 \mathrm{~d}-1$ \\
3 & $5.252993 \mathrm{~d}-1$ & $1.875407 \mathrm{~d}-1$ \\
4 & $4.285544 \mathrm{~d}-1$ & $2.024306 \mathrm{~d}-1$ \\
5 & $3.417059 \mathrm{~d}-1$ & $2.142341 \mathrm{~d}-1$ \\
\hline
\end{tabular}

Table 2. Computed values $C$ and $\sigma$ for different $b_{\gamma} . T=100$.

adapted to solve this problem and it is difficult to find theoretical studies that validate them, therefore to design and analyse innovative numerical procedures is really important.

In this study, an important issue in the modelization of cell population balance models has been addressed: the evolution of the size-distribution. We have proposed a suitable scheme to attain the solution of this model which allows us to analyse and approximate the stable size distribution if it exists. We have shown the behaviour of the steady-state distribution in two experiments. The first one aimed corroborating its independence of the initial size distribution. The second one at exploring its behaviour when the division rate changes. We have observed different stable size distributions for each problem and a clear influence on its maximum, the point at which this maximum is reached and the domain of significant densities.

The convergence of the numerical method was analysed: second order of convergence under suitable smoothness hypotheses.

\section{A. Convergence Analysis}

In this appendix, we carry out the convergence analysis of the scheme introduced in Section 2. From now on, $C$ will denote a positive constant which is independent of $k, n(0 \leq n \leq N)$ and $j(0 \leq j \leq J+1)$; $C$ possibly has different values in different places. We denote the maximum norm of $\mathbf{v}=\left(v_{0}, v_{1}, \ldots, v_{J+1}\right)$ with $\|\mathbf{v}\|_{\infty}$.

If $u$ is the solution to problem (1.1)-(1.3), we define

$$
\mathbf{u}^{n}=\left(u_{0}^{n}, u_{1}^{n}, \ldots, u_{J+1}^{n}\right), u_{j}^{n}=u\left(x_{j}, t^{n}\right), 0 \leq j \leq J+1,0 \leq n \leq N
$$

The local discretization error, $\boldsymbol{\tau}^{n+1}=\left(\tau_{0}^{n+1}, \tau_{1}^{n+1}, \ldots, \tau_{J+1}^{n+1}\right), 0 \leq n \leq N-1$, is given by

$$
\tau_{j+1}^{n+1}=\frac{1}{k}\left(u_{j+1}^{n+1}-\exp \left\{-\frac{k}{2}\left(\mu^{*}\left(x_{j}\right)+\mu^{*}\left(x_{j+1}\right)\right)\right\}\left(u_{j}^{n}+k \mathcal{Q}_{k}^{j}\left(\mathbf{b} \cdot \mathbf{P}^{j} \cdot \mathbf{u}^{n}\right)\right)-k \mathcal{Q}_{k}^{j+1}\left(\mathbf{b} \cdot \mathbf{P}^{j+1} \cdot \mathbf{u}^{n+1}\right)\right)
$$

$0 \leq j \leq J-1, \tau_{0}^{n+1}=\tau_{J+1}^{n+1}=0$

First of all, note that the magnitude of $J$ is not determined with respect to $k$. However, we prove the convergence of the composite quadrature rule over such a grid.

Lemma 1 Let $f$ be two times continuously differentiable, $\left\{x_{j}\right\}_{j=0}^{J+1}$ a nonuniform grid satisfying $x_{j+1}-x_{j} \leq C k, 0 \leq j \leq J, x_{0}=0$, $x_{J+1}=1$; and denote with $f_{l}=f\left(x_{l}\right), 0 \leq I \leq J+1$ the grid values of the function $f$ and with $\mathcal{Q}_{k}^{\prime}, 0 \leq I \leq J$, the composite trapezoidal quadrature rules given in (2.9). Then, as $k \rightarrow 0$, the following estimates hold

$$
\left|\int_{x_{l}}^{1} f(\sigma) d \sigma-\mathcal{Q}_{k}^{\prime}(\mathbf{f})\right|=O\left(k^{2}\right), \quad 0 \leq l \leq J
$$

Proof: From (2.9), we obtain

$$
\int_{x_{l}}^{1} f(\sigma) d \sigma-\mathcal{Q}_{k}^{\prime}(\mathbf{f})=\sum_{j=1}^{J}\left(\int_{x_{j}}^{x_{j+1}} f(\sigma) d \sigma-\frac{x_{j+1}-x_{j}}{2}\left(f\left(x_{j}\right)+f\left(x_{j+1}\right)\right)\right),
$$


$0 \leq I \leq J$. Taking into account the convergence properties of the trapezoidal cuadrature rule,

$$
\int_{x_{j}}^{x_{j+1}} f(\sigma) d \sigma-\frac{x_{j+1}-x_{j}}{2}\left(f\left(x_{j}\right)+f\left(x_{j+1}\right)\right)=-\frac{\left(x_{j+1}-x_{j}\right)^{3}}{12} f^{\prime \prime}\left(\xi_{j}\right), \xi_{j} \in\left[x_{j}, x_{j+1}\right],
$$

we have

$$
\int_{x_{l}}^{1} f(\sigma) d \sigma-\mathcal{Q}_{k}^{\prime}(\mathbf{f})=-\sum_{j=l}^{J} \frac{\left(x_{j+1}-x_{j}\right)^{3}}{12} f^{\prime \prime}\left(\xi_{j}\right)
$$

$0 \leq l \leq J$. Thus

$$
\left|\int_{x_{l}}^{1} f(\sigma) d \sigma-\mathcal{Q}_{k}^{\prime}(\mathbf{f})\right| \leq C \sum_{j=l}^{J}\left(x_{j+1}-x_{j}\right)^{3} \leq C k^{2} \sum_{j=l}^{J}\left(x_{j+1}-x_{j}\right)=C\left(1-x_{l}\right) k^{2} .
$$

And the estimative (A.2) holds.

Lemma 2 Let $g$ be three times continuously differentiable, functions $\mu, b \cdot P$ and $u$ be two times continuously differentiable. Then, as $k \rightarrow 0$, the following estimates hold for the local discretization error (A.1)

$$
\left\|\boldsymbol{\tau}^{n+1}\right\|_{\infty}=O\left(k^{2}\right), \quad 0 \leq n \leq N-1
$$

Proof: From (A.1), we obtain

$$
\begin{aligned}
\left|\tau_{j+1}^{n+1}\right| & \leq \frac{1}{k}\left|u_{j+1}^{n+1}-u\left(x\left(t^{n+1} ; t^{n}, x_{j}\right), t^{n+1}\right)\right| \\
& +\frac{1}{k}\left|u\left(x\left(t^{n+1} ; t^{n}, x_{j}\right), t^{n+1}\right)-k \mathcal{Q}_{k}^{j+1}\left(\mathbf{b} \cdot \mathbf{P}^{j+1} \cdot \mathbf{u}^{n+1}\right)-\exp \left\{-\frac{k}{2}\left(\mu^{*}\left(x_{j}\right)+\mu^{*}\left(x_{j+1}\right)\right)\right\}\left(u_{j}^{n}+k \mathcal{Q}_{k}^{j}\left(\mathbf{b} \cdot \mathbf{P}^{j} \cdot \mathbf{u}^{n}\right)\right)\right|
\end{aligned}
$$

$0 \leq j \leq J-1,0 \leq n \leq N-1$. The smoothness of $u$ and $g$, and the local error estimate for the modified Euler method [1] allow us to conclude

$$
\left|u_{j+1}^{n+1}-u\left(x\left(t^{n+1} ; t^{n}, x_{j}\right), t^{n+1}\right)\right| \leq C k^{3}
$$

In addition, we can use (2.4) to bound the second term on the right-hand side of (A.8) as

$$
\begin{aligned}
& \left|u\left(x\left(t^{n+1} ; t^{n}, x_{j}\right), t^{n+1}\right)-k \mathcal{Q}_{k}^{j+1}\left(\mathbf{b} \cdot \mathbf{P}^{j+1} \cdot \mathbf{u}^{n+1}\right)-\exp \left\{-\frac{k}{2}\left(\mu^{*}\left(x_{j}\right)+\mu^{*}\left(x_{j+1}\right)\right)\right\}\left(u_{j}^{n}+k \mathcal{Q}_{k}^{j}\left(\mathbf{b} \cdot \mathbf{P}^{j} \cdot \mathbf{u}^{n}\right)\right)\right| \\
& \leq\left|u_{j}^{n}\right|\left|\exp \left\{-\int_{t^{n}}^{t^{n+1}} \mu^{*}\left(x\left(\tau ; t^{n}, x_{j}\right)\right) d \tau\right\}-\exp \left\{-\frac{k}{2}\left(\mu^{*}\left(x_{j}\right)+\mu^{*}\left(x_{j+1}\right)\right)\right\}\right| \\
& +\mid 2 \int_{t^{n}}^{t^{n+1}} \exp \left\{-\int_{\tau}^{t^{n+1}} \mu^{*}\left(x\left(s ; t^{n}, x_{j}\right)\right) d s\right\}\left(\int_{x\left(\tau ; t^{n}, x_{j}\right)}^{1} b(\sigma) P\left(x\left(\tau ; t^{n}, x_{j}\right), \sigma\right) u(\sigma, \tau) d \sigma\right) d \tau \\
& -k\left(\exp \left\{-\frac{k}{2}\left(\mu^{*}\left(x_{j}\right)+\mu^{*}\left(x_{j+1}\right)\right)\right\} \mathcal{Q}_{k}^{j}\left(\mathbf{b} \cdot \mathbf{P}^{j} \cdot \mathbf{u}^{n}\right)+\mathcal{Q}_{k}^{j+1}\left(\mathbf{b} \cdot \mathbf{P}^{j+1} \cdot \mathbf{u}^{n+1}\right)\right) \mid
\end{aligned}
$$

Thus, we use the regularity of functions $\mu, b \cdot P$ and $g$, the convergence properties of the trapezoidal cuadrature rule and the modified Euler method to obtain

$$
\begin{aligned}
& \left|\exp \left\{-\int_{t^{n}}^{t^{n+1}} \mu^{*}\left(x\left(\tau ; t^{n}, x_{j}\right)\right) d \tau\right\}-\exp \left\{-\frac{k}{2}\left(\mu^{*}\left(x_{j}\right)+\mu^{*}\left(x_{j+1}\right)\right)\right\}\right| \\
& \leq\left|\exp \left\{-\int_{t^{n}}^{t^{n+1}} \mu^{*}\left(x\left(\tau ; t^{n}, x_{j}\right)\right) d \tau\right\}-\exp \left\{-\frac{k}{2}\left(\mu^{*}\left(x_{j}\right)+\mu^{*}\left(x\left(t^{n+1} ; t^{n}, x_{j}\right)\right)\right)\right\}\right| \\
& \quad+\exp \left\{-\frac{k}{2} \mu^{*}\left(x_{j}\right)\right\}\left|\exp \left\{-\frac{k}{2} \mu^{*}\left(x\left(t^{n+1} ; t^{n}, x_{j}\right)\right)\right\}-\exp \left\{-\frac{k}{2} \mu^{*}\left(x_{j+1}\right)\right\}\right| \\
& \leq C\left(k^{3}+k\left|\mu^{*}\left(x\left(t^{n+1} ; t^{n}, x_{j}\right)\right)-\mu^{*}\left(x_{j+1}\right)\right|\right) \leq C k^{3} .
\end{aligned}
$$


With respect to the second term on the right-hand side of (A.10),

$$
\begin{aligned}
& \mid \begin{array}{l}
\mid 2 \int_{t^{n}}^{t^{n+1}} \exp \left\{-\int_{\tau}^{t^{n+1}} \mu^{*}\left(x\left(s ; t^{n}, x_{j}\right)\right) d s\right\}\left(\int_{x\left(\tau ; t^{n}, x_{j}\right)}^{1} b(\sigma) P\left(x\left(\tau ; t^{n}, x_{j}\right), \sigma\right) u(\sigma, \tau) d \sigma\right) d \tau \\
-k\left(\exp \left\{-\frac{k}{2}\left(\mu^{*}\left(x_{j}\right)+\mu^{*}\left(x_{j+1}\right)\right)\right\} \mathcal{Q}_{k}^{j}\left(\mathbf{b} \cdot \mathbf{P}^{j} \cdot \mathbf{u}^{n}\right)+\mathcal{Q}_{k}^{j+1}\left(\mathbf{b} \cdot \mathbf{P}^{j+1} \cdot \mathbf{u}^{n+1}\right)\right) \mid \\
\leq 2 \mid \int_{t^{n}}^{t^{n+1}} \exp \left\{-\int_{\tau}^{t^{n+1}} \mu^{*}\left(x\left(s ; t^{n}, x_{j}\right)\right) d s\right\}\left(\int_{x\left(\tau ; t^{n}, x_{j}\right)}^{1} b(\sigma) P\left(x\left(\tau ; t^{n}, x_{j}\right), \sigma\right) u(\sigma, \tau) d \sigma\right) d \tau \\
\quad-\frac{k}{2}\left(\exp \left\{-\int_{t^{n}}^{t^{n+1}} \mu^{*}\left(x\left(s ; t^{n}, x_{j}\right)\right) d s\right\}\left(\int_{x_{j}}^{1} b(\sigma) P\left(x_{j}, \sigma\right) u\left(\sigma, t^{n}\right) d \sigma\right)\right. \\
+k \mid \exp \left\{-\int_{t^{n}}^{1} \mu^{*}\left(x\left(s ; t^{n}, x_{j}\right)\right) d s\right\}\left(\int_{x_{j}}^{1} b(\sigma) P\left(x_{j}, \sigma\right) u\left(\sigma, t^{n}\right) d \sigma\right) \\
\quad-\exp \left\{-\frac{k}{2}\left(\mu^{*}\left(x_{j}\right)+\mu^{*}\left(x_{j+1}\right)\right)\right\} \mathcal{Q}_{k}^{j}\left(\mathbf{b} \cdot \mathbf{P}^{j} \cdot \mathbf{u}^{n}\right) \mid \\
+k\left|\int_{x\left(t^{n+1} ; t^{n}, x_{j}\right)}^{1} b(\sigma) P\left(x\left(t^{n+1} ; t^{n}, x_{j}\right), \sigma\right) u\left(\sigma, t^{n+1}\right) d \sigma-\mathcal{Q}_{k}^{j+1}\left(\mathbf{b} \cdot \mathbf{P}^{j} \cdot \mathbf{u}^{n+1}\right)\right|
\end{array}
\end{aligned}
$$

The first term on the right-hand side of $(\mathrm{A} .12)$ is $O\left(k^{3}\right)$ as a result of the properties of the local error for the trapezoidal quadrature rule. With respect to the second term on the right-hand side of (A.12), we have

$$
\begin{aligned}
\left|\exp \left\{-\int_{t^{n}}^{t^{n+1}} \mu^{*}\left(x\left(s ; t^{n}, x_{j}\right)\right) d s\right\}\left(\int_{x_{j}}^{1} b(\sigma) P\left(x_{j}, \sigma\right) u\left(\sigma, t^{n}\right) d \sigma\right)-\exp \left\{-\frac{k}{2}\left(\mu^{*}\left(x_{j}\right)+\mu^{*}\left(x_{j+1}\right)\right)\right\} \mathcal{Q}_{k}^{j}\left(\mathbf{b} \cdot \mathbf{P}^{j} \cdot \mathbf{u}^{n}\right)\right| \\
\leq\left|\exp \left\{-\int_{t^{n}}^{t^{n+1}} \mu^{*}\left(x\left(s ; t^{n}, x_{j}\right)\right) d s\right\}-\exp \left\{-\frac{k}{2}\left(\mu^{*}\left(x_{j}\right)+\mu^{*}\left(x_{j+1}\right)\right)\right\}\right|\left|\int_{x_{j}}^{1} b(\sigma) P\left(x_{j}, \sigma\right) u(\sigma, t) d \sigma\right| \\
\quad+\exp \left\{-\frac{k}{2}\left(\mu^{*}\left(x_{j}\right)+\mu^{*}\left(x_{j+1}\right)\right)\right\}\left|\int_{x_{j}}^{1} b(\sigma) P\left(x_{j}, \sigma\right) u\left(\sigma, t^{n}\right) d \sigma-\mathcal{Q}_{k}^{j}\left(\mathbf{b} \cdot \mathbf{P}^{j} \cdot \mathbf{u}^{n}\right)\right| .
\end{aligned}
$$

Note that the numerical grid is computed by the modified Euler method and then satisfies the hypotheses of Lemma 1 [1]. Thus, taking into account the assumed regularity of the vital functions and solution, the second order convergence of the composite quadrature rule (Lemma 1) and (A.11), we conclude that the previous term is $O\left(k^{2}\right)$.

Finally, the third term on the right-hand side of (A.12) is bounded as follows

$$
\begin{aligned}
& \left|\int_{x\left(t^{n+1} ; t^{n}, x_{j}\right)}^{1} b(\sigma) P\left(x\left(t^{n+1} ; t^{n}, x_{j}\right), \sigma\right) u\left(\sigma, t^{n+1}\right) d \sigma-\mathcal{Q}_{k}^{j+1}\left(\mathbf{b} \cdot \mathbf{P}^{j} \cdot \mathbf{u}^{n+1}\right)\right| \\
& \leq\left|\int_{x\left(t^{n+1} ; t^{n}, x_{j}\right)}^{x_{j+1}} b(\sigma) P\left(x\left(t^{n+1} ; t^{n}, x_{j}\right), \sigma\right) u\left(\sigma, t^{n+1}\right) d \sigma\right|+\left|\int_{x_{j+1}}^{1} b(\sigma)\left(P\left(x\left(t^{n+1} ; t^{n}, x_{j}\right), \sigma\right)-P\left(x_{j+1}, \sigma\right)\right) u\left(\sigma, t^{n+1}\right) d \sigma\right| \\
& +\left|\int_{x_{j+1}}^{1} b(\sigma) P\left(x_{j+1}, \sigma\right) u\left(\sigma, t^{n+1}\right) d \sigma-\mathcal{Q}_{k}^{j+1}\left(\mathbf{b} \cdot \mathbf{P}^{j+1} \cdot \mathbf{u}^{n+1}\right)\right|
\end{aligned}
$$

And, we can conclude, as previously, that this term is $O\left(k^{2}\right)$.

Thus, we establish for the left term on (A.12)

$$
\begin{aligned}
\mid 2 \int_{t^{n}}^{t^{n+1}} \exp \{- & \left.\int_{\tau}^{t^{n+1}} \mu^{*}\left(x\left(s ; t^{n}, x_{j}\right)\right) d s\right\}\left(\int_{x\left(\tau ; t^{n}, x_{j}\right)}^{1} b(\sigma) P\left(x\left(\tau ; t^{n}, x_{j}\right), \sigma\right) u(\sigma, \tau) d \sigma\right) d \tau \\
& \quad k\left(\exp \left\{-\frac{k}{2}\left(\mu^{*}\left(x_{j}\right)+\mu^{*}\left(x_{j+1}\right)\right)\right\} \mathcal{Q}_{k}^{j}\left(\mathbf{b} \cdot \mathbf{P}^{j} \cdot \mathbf{u}^{n}\right)+\mathcal{Q}_{k}^{j+1}\left(\mathbf{b} \cdot \mathbf{P}^{j+1} \cdot \mathbf{u}^{n+1}\right)\right) \mid \leq C k^{3},
\end{aligned}
$$


and from (A.11), we observe that the right-hand side of (A.10) is $O\left(k^{3}\right)$. The substitution of this bound and (A.9) in (A.8) produces the estimate (A.7).

Now, we prove the convergence of the numerical method. We denote the error produced by the numerical approximation as

$$
\mathbf{E}^{n}=\left(E_{0}^{n}, \ldots, E_{J}^{n}, E_{J+1}^{n}\right), \quad E_{j}^{n}=u_{j}^{n}-U_{j}^{n}, \quad 0 \leq j \leq J+1
$$

$0 \leq n \leq N$. Note that $u_{j}^{n}$ are the nodal values of the theoretical solution and $U_{j}^{n}$ are the numerical approximations obtained by means of the numerical method.

Theorem 3 Assuming the hypotheses of Lemma 2, if $\left\|\mathbf{E}^{0}\right\|_{\infty}=O\left(k^{2}\right)$, as $k \rightarrow 0$, then

$$
\left\|\mathbf{E}^{n}\right\|_{\infty}=O\left(k^{2}\right), \quad 0 \leq n \leq N,
$$

as $k \rightarrow 0$.

Proof: From equations (A.1) and (2.7), we have

$$
E_{j+1}^{n+1}=\exp \left\{-\frac{k}{2}\left(\mu^{*}\left(x_{j}\right)+\mu^{*}\left(x_{j+1}\right)\right)\right\}\left(E_{j}^{n}+k \mathcal{Q}_{k}^{j}\left(\mathbf{b} \cdot \mathbf{P}^{j} \cdot \mathbf{E}^{n}\right)\right)+k \mathcal{Q}_{k}^{j+1}\left(\mathbf{b} \cdot \mathbf{P}^{j+1} \cdot \mathbf{E}^{n+1}\right)+k \tau_{j+1}^{n+1},
$$

$0 \leq j \leq J-1,0 \leq n \leq N-1$.

Therefore, taking into account the smoothness properties of the functions $\mu^{*}$ and $b$ we arrive at,

$$
\left|E_{j+1}^{n+1}\right| \leq(1+C k)\left|E_{j}^{n}\right|+C k\left(\left\|\mathbf{E}^{n}\right\|_{\infty}+\left\|\mathbf{E}^{n+1}\right\|_{\infty}\right)+k\left|\tau_{j+1}^{n+1}\right|
$$

$0 \leq j \leq J-1,0 \leq n \leq N-1$, and then

$$
\left\|\mathbf{E}^{n+1}\right\|_{\infty} \leq(1+C k)\left\|\mathbf{E}^{n}\right\|_{\infty}+C k\left\|\mathbf{E}^{n+1}\right\|_{\infty}+k\left\|\boldsymbol{\tau}^{n+1}\right\|_{\infty}
$$

$0 \leq n \leq N-1$. By means of the discrete Gronwall's lemma, we arrive at

$$
\left\|\mathbf{E}^{n}\right\|_{\infty} \leq C\left\{\left\|\mathbf{E}^{0}\right\|_{\infty}+\sum_{l=1}^{n} k\left\|\boldsymbol{\tau}^{\prime}\right\|_{\infty}\right\}
$$

$1 \leq n \leq N$. Now, the estimative holds from (A.7). .

\section{Acknowledgements}

This work was supported in part by projects MTM2014-56022-C2-2-P and MTM2017-85476-C2-1-P:of the Spanish Ministerio de Economía y Competitividad and European FEDER Funds, and by project VA041P17 of the Junta de Castilla y León and European FEDER Funds.

\section{References}

1. Angulo O, López-Marcos JC. Numerical schemes for size-structured population equations. Mathematical Biosciences 1999; 157: 169-188.

2. Angulo O, López-Marcos JC, López-Marcos MA. A semi-Lagrangian method for a cell population model in a dynamical environment. Mathematical and Computer Modelling 2013; 57: 1860-1866

3. Angulo O, López-Marcos JC, López-Marcos MA. A second-order method for the numerical integration of a size-structured cell population model. Abstract and Applied Analysis 2015; 549168: 1-8. DOI:10.1155/2015/549168. 
4. Arino O. A survey of structured cell population dynamics. Acta Biotheoretica 1995; 43: 3-25.

5. Bell GI, Anderson EC. Cell growth and division: I. a mathematical model with applications to cell volume distributions in mammalian suspension cultures. Biophysical Journal 1967; 7: 329-351.

6. Borges R, Calsina A, Cuadrado S. Oscillations in a molecular structured cell population model. Nonlinear Analysis: Real Word Applications 2011; 12: 1911-1922.

7. Cushing JM. An Introduction to Structured Population Dynamics. CMB-NSF Regional Conference Series in Applied Mathematics 71 , SIAM: Philadelphia; 1998

8. Diekmann O, Heijmans HJAM, Thieme HR. On the stability of the cell size distribution. Journal of Mathematical Biology 1984; 19: 227-248

9. Fadda S, Cincotti A, Cao G. A Novel Population Balance Model to Investigate the Kinetics of In Vitro Cell Proliferation: Part I. Model Development. Biotechnology and Bioengineering 2012; 109: 772-781.

10. Fadda S, Cincotti A, Cao G. A Novel Population Balance Model to Investigate the Kinetics of In Vitro Cell Proliferation: Part II. Numerical Solution, Parameters, Determination, and Model Outcomes. Biotechnology and Bioengineering 2012; 109: $782-796$.

11. Fredrickson AG, Ramkrishna D, Tsuchiya HM. Statistics and dynamics of procaryotic cell populations. Mathematical Biosciences 1967; 1: $327-374$

12. Gyllenberg M, Webb GF. Asynchronous Exponential Growth of Semigroups of Nonlinear Operators, J. Math. Anal. Appl. 1992; 167: 443-467.

13. Heijmans HJAM. On the stable size distribution of populations reproducing by fission into two unequal parts. Mathematical Biosciences 1984; 72: 19-50

14. Kato N. A general model of size-dependent population dynamics with nonlinear growth rate. J. Math. Anal. Appl. 2004; 297: 234-256.

15. Ito K, Kappel F, Peichl G. A fully discretized approximation scheme for size-structured population models. SIAM Journal of Numerical Analysis 1991; 28: 923-954.

16. Lasota A, Mackey MC. Probabilistic Properties of Deterministic Systems. Cambridge University Press: London; 1985.

17. Metz JAJ, Diekmann O (Eds.). The Dynamics of Physiologically Structured Populations. In: Lect. Notes Biomath. 68, Springer-Verlag: New York; 1986

18. Perthame B. Transport Equations in Biology. Birkhuser: Basel, Switzerland; 2007.

19. Ramkrishna D. Statistical models of cell populations. In: Advances in Biochemical Engineering. 11, Springer: Berlin; 1979; 1-47,

20. Ramkrishna D, Singh MR. Population Balance Modeling: Current Status and Future Prospects. Annual Review of Chemical and Biomolecular Engineering. 2014; 5: 123-146

21. Randolph AD. A population balance for countable entities. The Canadian Journal of Chemical Engineering. 1964; 42: $280-281$.

22. Spetsieris K, Zygourakis K. Single-Cell Behavior and Population Heterogeneity: Solving an Inverse Problem to Compute the Intrinsic Physiological State Functions. Journal of Biotechnology 2012; 158: 80-90. 